\title{
Empirical system of image enhancement for digital microscopic pneumonia bacteria images
}

\author{
Ganesh Kumar $\mathrm{T}^{1}$, Asha $\mathrm{V}^{2}$, Manish $\mathrm{TI}^{3}$, Muthulakshmi $\mathrm{G}^{2}$ \\ School of Computing Science and Engineering, Galgotias University, NCR, Delhi, India. \\ tganeshphd@yahoo.com
}

\begin{abstract}
In this paper the image processing method is used to enhance the pneumonia bacteria images. This paper recognized the bacteria images based on two domains. The enhancement techniques used for bacteria image enhancement were median filter, wiener filter, single scale retinex and multiscale retinex. Image enhancement has a very important role in digital image processing. The median and wiener filters were used for grayscale image enhancement. Then single scale retinex and multiscale retinex were used for color image enhancement. Based on performance metrics identified median filter is suitable for bacteria images in grayscale image enhancement and multiscale retinex is suitable for bacteria color image enhancement (Tab. 2, Fig. 8, Ref. 21). Text in PDF www.elis.sk.

KEY WORDS: Pneumonia bacteria, image processing, image enhancement, median filter, wiener filter, single scale retinex, multiscale retinex.
\end{abstract}

\section{Introduction}

Bioinformatics is the application of computer techniques to access biological information. Computers are utilized to assemble, store, analyze and incorporate genetic and biological information, which can then be connected to quality based medication revelation and advancement. The publicly available genomic information ensuing from the Human Genome has precipitated the need for Bioinformatics capacities. The objective of this work is to determine the sequence of the whole human genome around three billion base sets. The investigations of Bioinformatics, which is the merging of biology with computer science, is crucial for the utilization of genomic data in understanding human diseases and in the recognition of new molecular targets for drug discovery. In recognition of this, several universities, government research centres and pharmaceutical companies have formed bioinformatics teams, consisting of computational biologists and bioinformatics computer scientists. Such teams are going to unravel the mass of data generated by massive scale sequencing efforts currently in laboratories around the world.The term bioinformatics did not mean what it means these days and refers to the study of knowledge processes in organic phenomenon systems. This definition places bioinformatics as a field parallel to physical science or biochemistry $(1,2,3)$.

${ }^{1}$ Galgotias University, NCR, Delhi, India, ${ }^{2}$ Manonmaniam Sundaranar University, Tirunelveli, India, and ${ }^{3}$ Adi Sankara Institute of Engineering and Technology, Kerala, India

Address for correspondence: Dr. Ganesh Kumar T, School of Computing Science and Engineering, Galgotias University, NCR, Delhi, India.

\section{Literature review}

It is an interdisciplinary field that creates techniques and software tools for comprehension of biological information. Bioinformatics currently entails the creation and advancement of databases, algorithms, process and applied mathematics techniques, and theory to resolve formal and sensible issues arising from the management and analysis of biological knowledge. Bioinformatics is the name given to these mathematical and computing approaches used to glean understanding of biological processes. Image enhancement is used to improve the digital quality of image. It is used to improve the poor quality of image that is too used to improve bad quality of picture into good picture or image (4). A fuzzy rule-based algorithm used for the enhancement of high intensity images. The fuzzy sets are modelled using Gaussian membership functions and experiments are using Mamdani and Larsen fuzzy inferences (5). A method of Gaussian distribution mapping which also keeps the information laid on the pattern of the histogram on the original image (6). In the first algorithm, called Automatic Segmentation and Histogram Stretching(ASHS) the original image is strongly improved by a Local Contrast Enhancement(LCE) algorithm (7). The Moving Median Filter is similar to the moving average filter, except that it produces as output at a pixel the median (8). The SVD-based techniques enhance the low contrast images presented (9). An efficient framework for color image enhancement in the compressed wavelet domain. The proposed method is able to enhance a wavelet-coded image computationally efficiently with high image quality and less noise or other artifacts presented (10). A novel infrared-to-visible image fusion algorithm for enhancing contrast is proposed. The quality of the 
fusion images was improved for better differentiability in terms of contrast and features of the targets (11). Canny filter with scale multiplication and colony optimization techniques $(12,13)$. The Gaussian noise can be reduced using a spatial filter and frequency filter (14). A pipelined approach including histogram averaging, color channel stretching and re-mapping is developed (15). Bilateral filtering technique is smoothing an image while preserving edges, by means of a nonlinear arrangement of nearby image value (16). The Cometary Coma Image Enhancement Facility (CometCIEF) allows the user to enhance FITS images using five advanced image enhancement techniques which were not previously available as an open source. The enhancement process can then be downloaded as FITSimages (17). An image enhancement method is the wavelet-based contourlet transform (WBCT) with cycle translation methods (18).

\section{Material and methods}

\section{Material}

Pneumonia bacteria/Pseudomonas aeruginosa

Pneumonia bacteria/Pseudomonas aeruginosa is common Gram-negative rod shaped bacteria that causes disease in plants, animals and humans. A species of significant medical importance, $\mathrm{P}$. aeruginosa is an ideal multidrug resistant (MDR) pathogen that is recognised for its ubiquity. It has developed an antibiotic resis- tance mechanisms, and is associated with severe illnesses - mainly nosocomial infections such as ventilator pneumonia and different sepsis syndromes. P. aeruginosa is among the Gram-negative bacilli commonly isolated from the exoskeletons and/or droppings of the cosmopolitan peridomestic cockroaches - including P (Periplaneta) Americana and B (Blatella) germanica - which are often pervasive in households and hospital settings. Severe Pseudomonas infection regularly occurs in people in the hospital with weakened immune systems. Infections of the blood, pneumonia, and infections following surgery can lead to severe disease in these people. However, healthy people can have mild illness with Pseudomonas aeruginosa, mainly after exposure to water. Ear infections, especially in children and generalized skin rashes may occur after exposure to inadequately chlorinated swimming pools. In hospitals, where the most severe infections occur, Pseudomonas can be spread by the hands of healthcare workers or by equipment that gets contaminated and is not properly cleaned. Figure $1 \mathrm{a}-\mathrm{f}$ shows the digital microscopic pseudomonas aeruginosa image. These images were used for images enhancement.

\section{Methods}

\section{Median Filter}

Processing of the image causes some kind of noise reduction in the image. The median filter is a nonlinear digital filtering technique used to remove the noise. This filter is widely used in digi-
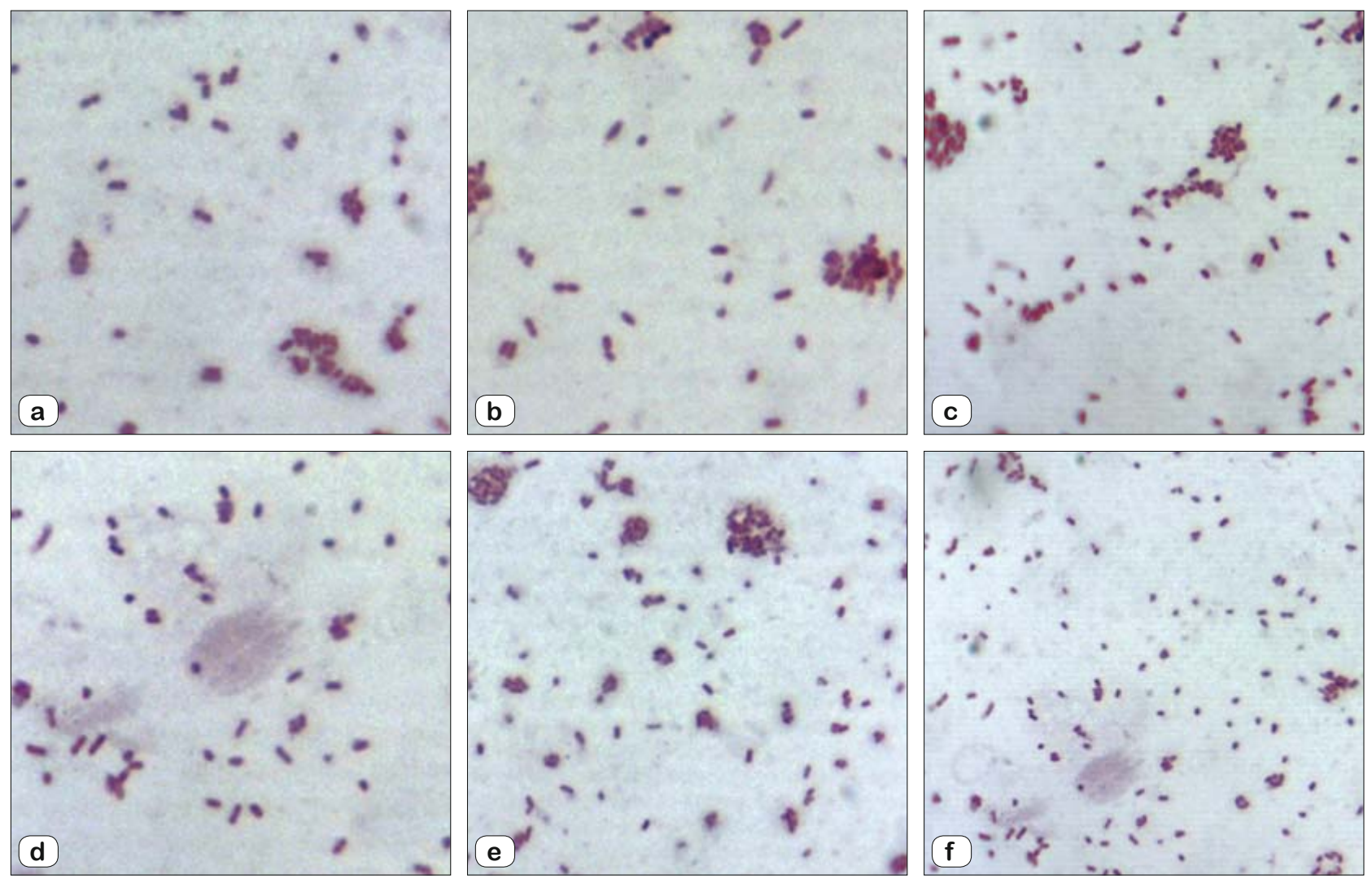

Fig. 1 (a-f). Digital Microscopic Pseudomonas Aeruginosa. 


\begin{tabular}{l|l|l|l|l|l|l} 
& & & & & & \\
\hline & 122 & 124 & 125 & 129 & 139 & \\
\hline & 121 & 123 & 125 & 126 & 134 & \\
\hline & 117 & 119 & 149 & 124 & 133 & \\
\hline & 118 & 114 & 118 & 122 & 132 & \\
\hline & 110 & 115 & 109 & 119 & 129 & \\
\hline & & & & & &
\end{tabular}

$$
\begin{array}{ll}
\text { Neighboured values: } & 114,117,119,122,123, \\
& 124,125,126,149 \\
\text { Median value: } & 123
\end{array}
$$

Fig. 2. Calculating the median value of a pixel neighbourhood. As can be seen, the central pixel value of $\mathbf{1 4 9}$ is rather unrepresentative of the surrounding pixels and is replaced with the median value: 123 . A $3 \times 3$ square neighbourhood is used here --- larger neighbourhoods will produce more severe smoothing.

tal image processing, under certain conditions, it preserves edges while removing noise (19). The median filter is to run through the signal entry by entry like pattern. The pattern of neighbours is called the "window", which slides, entry by entry through the entire digital image. For 1D image, the most common window is the first few preceding and following entries, whereas for 2D images, more complex window patterns are possible. If the window has an odd number of entries, then the median is simple to define: it is just the middle value after number sorts all the entries in the window. An even number of entries is more than one possible median (20). The median is calculated by first sorting all the pixel values from the surrounding neighbourhood into numerical order and then replacing the pixel being considered with the middle pixel value. Figure 2 illustrates an example calculation.

By calculating the median value of a neighbourhood rather than the mean filter, the median filter has two main advantages over the mean filter.

The median filter does not create new unrealistic pixel numeric values when the filter straddles an edge (21). Edge preservation properties - Median filtering is one kind of smoothing technique, as is linear Gaussian filtering. Edges are of critical importance to the visual appearance of images. It is particularly effective. Because of this, median filtering is very widely used in digital image processing (Fig. $3 \mathrm{a}-\mathrm{f}$ ).

\section{Wiener filter}

Wiener filtering technique is used in a wide range of applications such as linear prediction, signal restoration, channel equalisation, echo cancellation and system identification. The $r$ filter coefficients are calculated to minimise the average squared
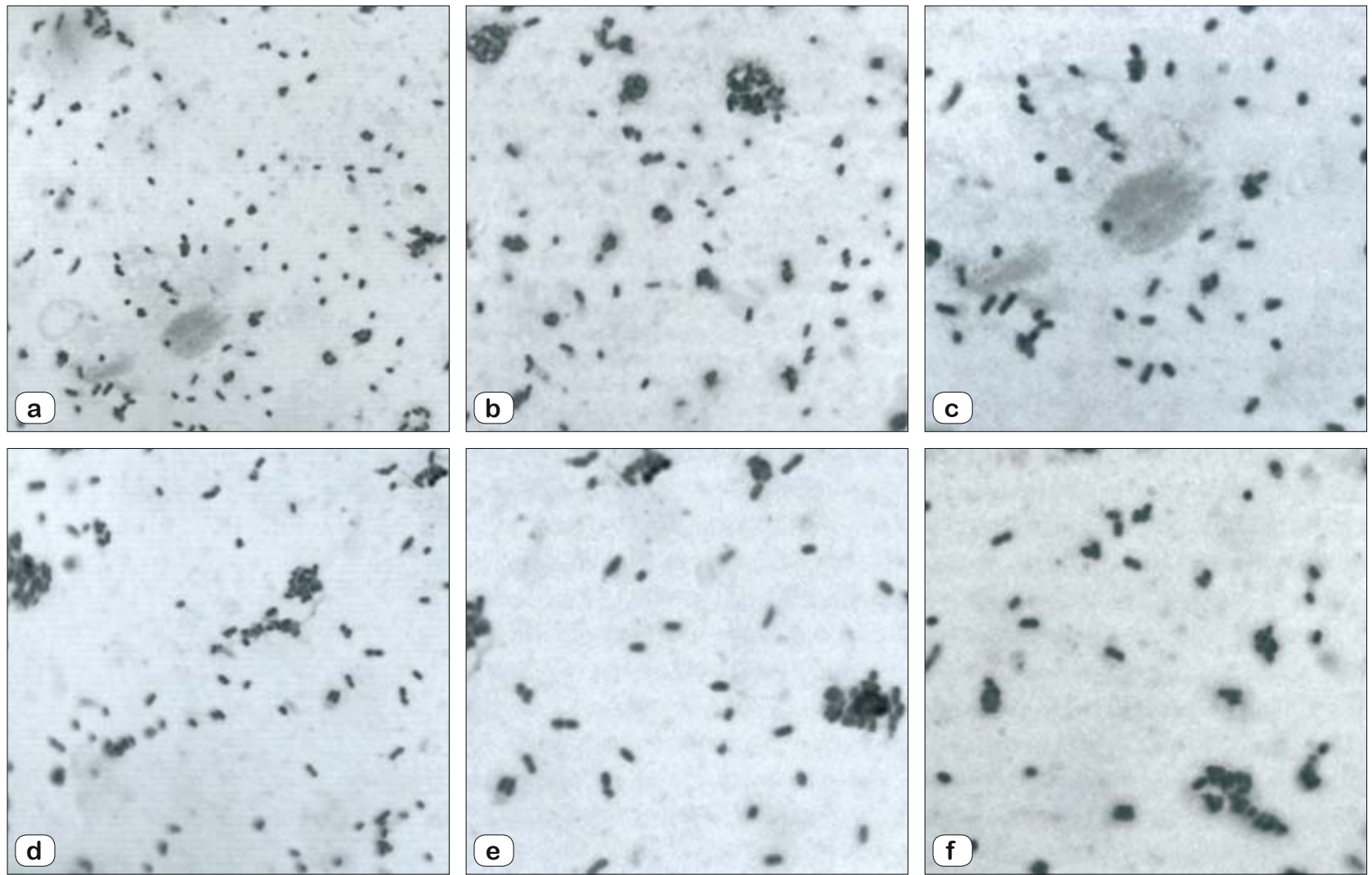

Fig. 3 (a-f). Median filter image enhancement. 
distance between the filter output and a desired image. The Wiener filtering technique assumes that the signals are stationary processes. A block-adaptive filter can be used for image that may be considered almost stationary over a relatively small block of samples. The application of Wiener filters in time-delay estimation, channel equalisation and additive noise reduction. The Wiener filtering executes an optimal trade off between inverse filtering and noise smoothing. It removes the additive noise and inverts the blurring simultaneously in an image. The Wiener filtering is optimal in terms of the mean square error. The Wiener filtering is a linear estimation of the original image. The approach consists of a stochastic framework. The orthogonality principle implies that the Wiener filter in Fourier domain can be expressed as follows:

$$
W\left(f_{1}, f_{2}\right)=\frac{H *\left(f_{1}, f_{2}\right) S_{x x}\left(f_{1}, f_{2}\right)}{\left|H\left(f_{1}, f_{2}\right)\right|^{2} S_{x x}\left(f_{1}, f_{2}\right)+S_{n n}\left(f_{1}, f_{2}\right)}
$$

Where $S_{x x}\left(f_{1}, f_{2}\right), S_{n n}\left(f_{1}, f_{2}\right)$ are the respective power spectra of the original image and the additive noise, and $\mathrm{H}\left(\mathrm{f}_{1}, \mathrm{f}_{2}\right)$ is the blurring filter. It is easy to see that the Wiener filter has two separate parts, an inverse filtering part and a noise smoothing part. It not only performs the deconvolution by inverse filtering (high pass filtering) but also removes the noise with a compression operation (low pass filtering). a) Implementation

The Wiener filter in practice estimates the power spectra of the original image and the additive noise. For white additive noise the power spectrum is equal to the variance of the noise. To assess the power spectrum of the original image many methods can be used. A direct estimate is the periodogram estimate of the power spectrum computed from the observation:

$$
S_{y y}^{p e r}=\frac{1}{N^{2}}\left[Y(k, l) Y(k, l)^{*}\right]
$$

Where $Y(k, l)$ is the DFT of the observation. The advantages of the estimate are that it can be implanted very easily without worrying about the singularity of the inverse filtering. Another estimate which leads to a cascade implementation of the inverse filtering and the noise smoothing is

$$
S_{x x}=\frac{S_{y y}-S_{n n}}{|H|^{2}}
$$

Which is a straight forward result of $S_{y y}=S_{n n}+S_{x x}|H|^{2}$ The power spectrum $S_{y y}$ is considered as estimated directly from the observation using the periodogram estimate. This estimate results in a cascade implementation of inverse filtering and noise smoothing:

$$
W=\frac{1}{H} \frac{S_{y y}^{p e r}-S_{n n}}{S_{y y}^{p e r}}
$$
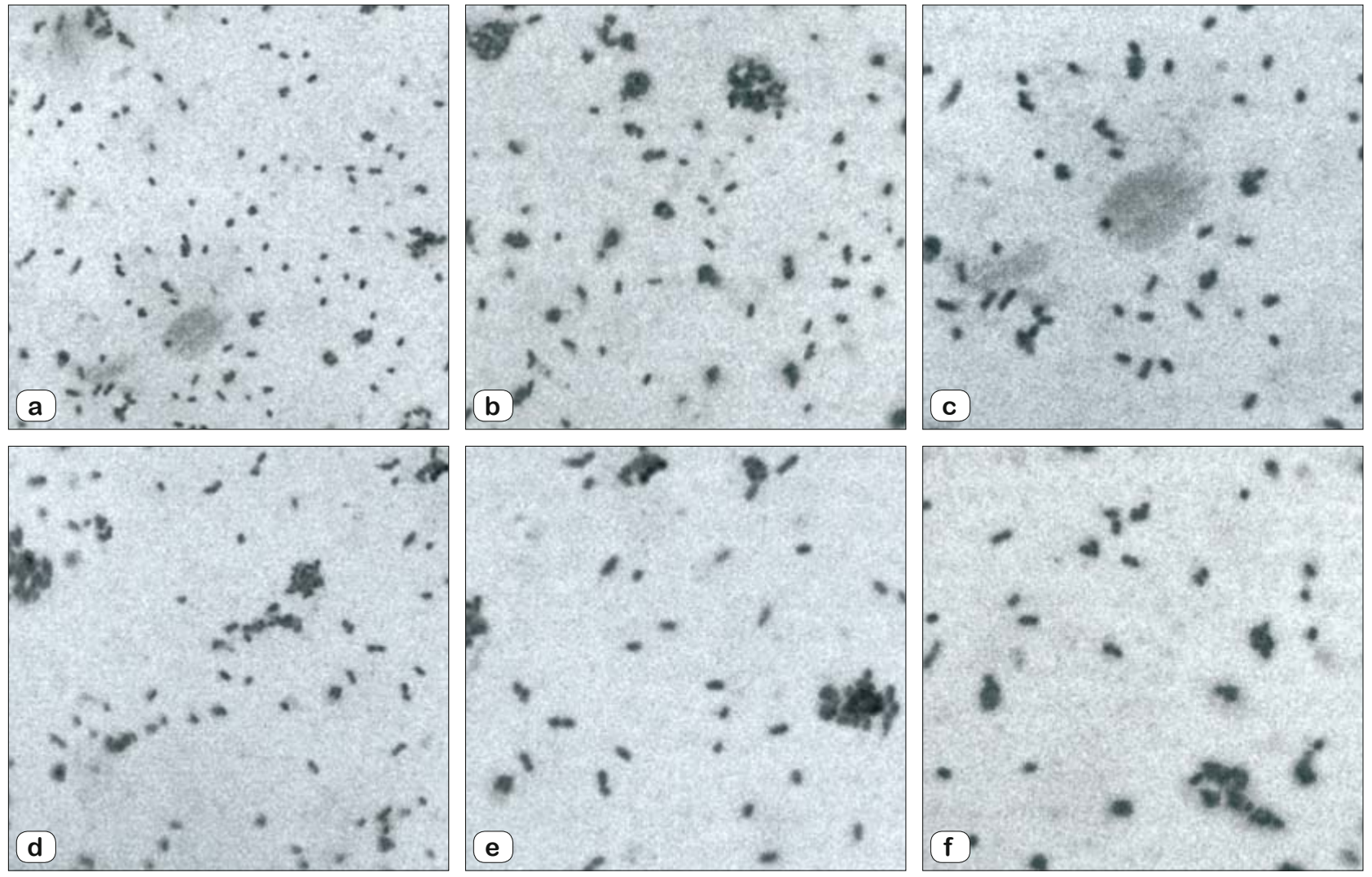

Fig. 4 (a-f). Wiener filter image enhancement result. 


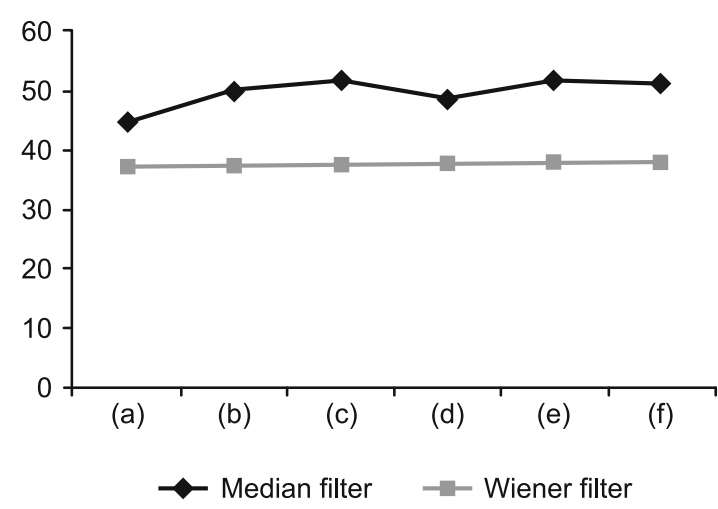

Fig. 5. Performance chart of the Grayscale filters.

The wiener filtering is applied to the image with a cascade implementation of the noise smoothing and inverse filtering (Figs 4 a-f and 5).

Single scale retinex for color enhancement

The Retinex is a general purpose image enhancement algorithmic rule that's used to increase the contrast, brightness and perceived sharpness of images primarily through dynamic range compression. The algorithm also simultaneously provides color constant output thus it removes the effects caused by different illuminants on a scene. It synthesizes contrast enhancement and color reliability by performing a non-linear spatial/spectral transform that mimics traits seen in the human vision system. The algorithm has been successfully applied to medical microscopic images.

\section{b) Retinex}

The Retinex is a member of the class of center surround functions where each output value is determined by the corresponding input value. It is called center value and its neighbourhood. It is called surround value. For the Retinex the center is defined as each pixel value and the surround is a Gaussian function. The arithmetic form of the single-scale Retinex (ssr) is given by

$$
R\left(x_{1}, x_{2}\right)=\alpha\left(\log \left(I\left(x_{1}, x_{2}\right)\right)-\log \left(I\left(x_{1}, x_{2}\right) * F\left(x_{1}, x_{2}\right)\right)\right)-\beta
$$

Where $I$ is the input microscopic image, $R$ is the output of the Retinex algorithm image, $\log$ is the natural logarithm function, $\alpha$ is a scaling(gain) factor and $\beta$ is an offset parameter. The "*" symbol represents convolution. $F$ is a Gaussian filter (surround or kernel) defined by

$$
\left.F\left(x_{1}, x_{2}\right)=k \exp \left[-x_{1}^{2}+x_{2}^{2}\right) / \sigma^{2}\right]
$$

Where $\sigma$ is the standard deviation of the filter, and $\mathrm{k}$ is a normalization factor for the Gaussian curve equal to 1 . The $\alpha, \beta$ and $\sigma$ parameters are determined empirically.

As is easily observed from the Retinex equation, the tallest processing pole is the convolution operation. Large Gaussian kernels, typically with $\sigma$ ranging from 50 to 120 are normally used to produce good single-scale Retinex performance, thus spatial domain convolution would be extremely time consuming and naturally turn to the well-known equivalence between convolution in the spatial domain and multiplication in the spatial-frequency domain.

$$
f(x, y)^{*} g(x, y) \Leftrightarrow F(\mu, v) G(\mu, v)
$$

where $F$ and $G$ are the spatial frequency domain representations of $f$ and $g$ respectively. The 2-dimensional $M \times N$ forward and inverse Discrete Fourier Transforms (DFT),

$$
\begin{aligned}
& F(\mu, v)=\frac{1}{M N} \sum_{x=0}^{M-1} \sum_{x=0}^{N-1} f(x, y) \exp [-j 2 \pi(\mu x / M+v y / N)] \\
& f(x, y)=\sum_{\mu=0}^{M-1} \sum_{v=0}^{N-1} F(\mu \mid, v) \exp [j 2 \pi(\mu x / M+v y / N)],
\end{aligned}
$$

To rewrite the Retinex equation as:

$R\left(x_{1}, x_{2}\right)=\alpha\left(\log \left(I\left(x_{1}, x_{2}\right)\right)-\log \left[F^{-1}\left(I^{\prime}(\mu, v) F^{\prime}(\mu, v)\right)\right]\right)-\beta$,

where $I^{\prime}(\mu, v)$ and $F^{\prime}(\mu, v)$ represents the DFTs of $I\left(x_{1}, x_{2}\right)$ and $F\left(x_{1}, x_{2}\right)$ respectively, and $F^{-1}$ represents the inverse DFT. The DFTs are computed using the well- known Fast Fourier Transform (FFTs) and by processing the 2-dimensional image transform by applying 1-dimensional FFTs first to the rows, and then to the columns of the image. DSPs are known for their ability to rapidly compute FFTs and the associated bit reversal required so this efficiency maps well into the equation above (Fig. $6 \mathrm{a}-\mathrm{f}$ ).

Multiscale retinex for color enhancement

A typical issue with color imagery- digital or analog- is that of successful capture of the dynamic range and colors seen through the viewfinder onto the gained image. As a rule, this picture is a poor version of the actual observed scene. The Single Scale Retinex indicates uncommon guarantee for element extend pressure however does not give great tonal interpretation. In fact, a distinctive trade-off inhibited by the scale of the surround function exists between dynamic range compression and tonal rendition, and one can be enhanced just at the cost of decreasing the other. This work in reducing some of these trade-offs by utilizing a multiscale retinex(MSR), i.e a retinex which joins a few SSR yields to a single output image which has good tonal retinal rendition. The tonal rendition, though, is still scene dependent to a specific extent. A portion of the consequence of applying the MSR to color images and compare our outcomes and different techniques for image enhancement.

The MSR can be compactly written as

$F(x, y)=\sum_{n=1}^{N} W_{n}\left\{\log \left[S_{i}(x, y)\right]-\log \left[S_{i}(x, y) * M_{n}(x, y)\right]\right\}$

Where the subscripts $i \in \mathrm{R}, \mathrm{G}, \mathrm{B}$ represent the three color bands, $\mathrm{N}$ is the number of scales being utilized, and $\mathrm{W}_{\mathrm{n}}$ are the weighting elements for the scales. The $M_{n}(x, y)$ are the encompass capacities given by

$$
M_{n}(x, y)=K_{n} \exp \left[-\left(x^{2}+y^{2}\right) / \sigma_{n}^{2}\right.
$$



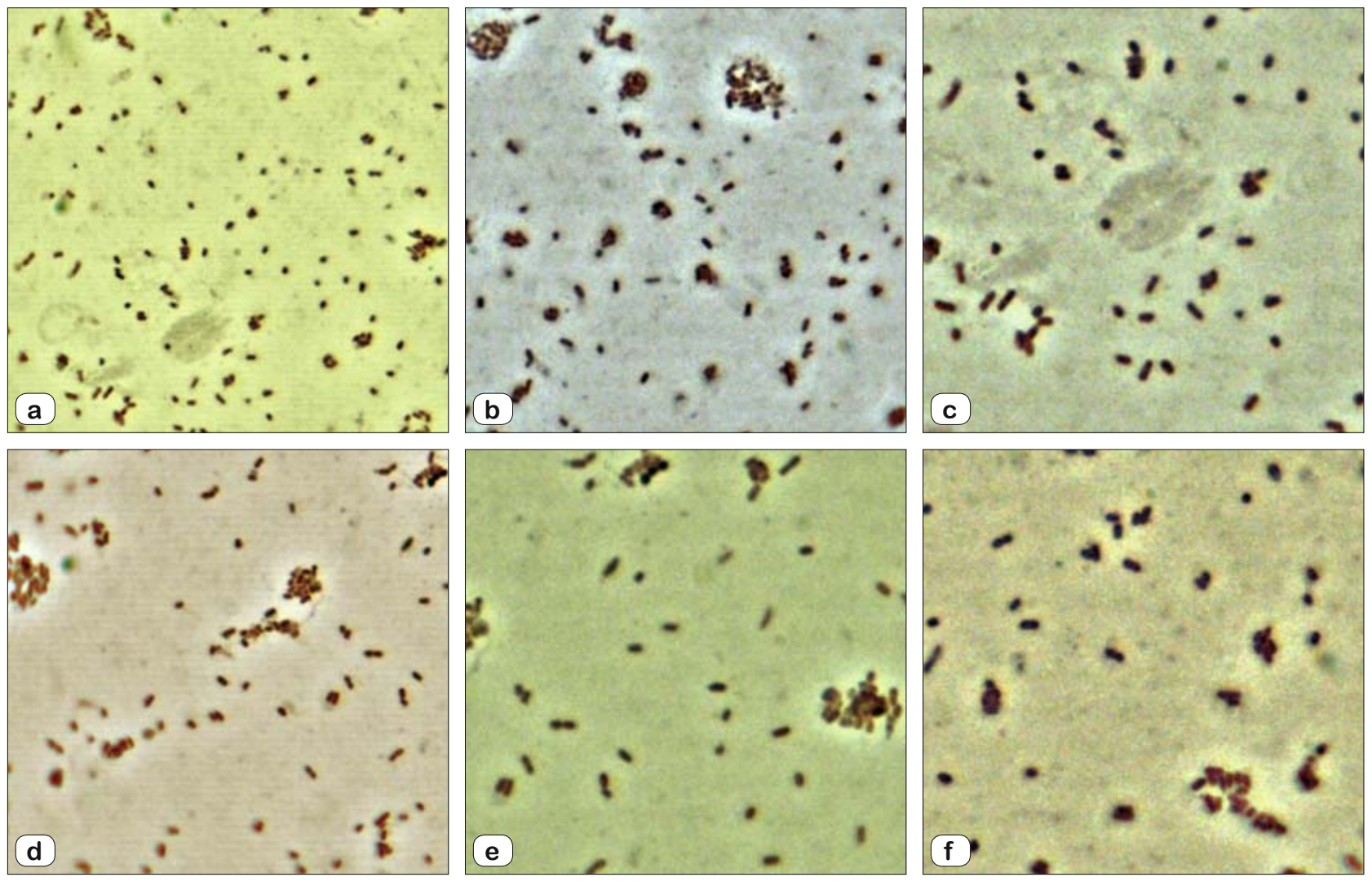

Fig. 6 (a-f). Single scale color image enhancement result.

Where the $\sigma_{n}$ are the standard deviations of the Gaussian distribution that decide the scale. The size of the scale decides the kind of data that the retinex gives: smaller scales providing more dynamic range compression, and larger scales providing more color constancy. The $\mathrm{K}_{\mathrm{n}}$ are selected so that $\iint F(x, y) d x d y=1$.

Each of the expressions inside the summation in Equation 1 represents an SSR.

The SSR has been previously defined to have the following characteristics and properties.

1) The function form of the surround is a Gaussian. 2) The palcement of the log function is AFTER surround formation. 3) The post-retinex signal processing is a "canonical" gain-offset rather than an automatic gain-offset. 4) There is a trade-off between dynamic range compression and tonal interpretation which is represented by the Gaussian surround space constant. A space consistent of 80 pixels was a sensible trade off between dynamic range compression and interpretation. 5) A single scale seemed incapable of simultaneously provinding sufficient dynamic range compression and tonal rendition. 6) Violations of the gray-world assumption led to retinexed images which were either " graed-out' locally or globally or, more rarely, suffered from color distortion.

The MSR joins the dynamic range compression of the small scale retinex with the tonal version of the large scale retinex to create a yield which incorporates both.
As stated above, the MSR still experiences turning grayout of uniform zones much as the SSR did. The favorable circumstances that the MSR has over the MSR has over the SSR is in the combintion of scales which give both element extend pressure and aggregate interpretation in the mean time. The overall result of the application of the MSR is still more saturated than human observation, giving the final image a "washes-out" apperance, but it preserves most of the detail in the scene. This "graying" of areas of constant intensity occurs because the retinex processing enhances each color band as a funtion of its surround. The smaller values in the weaker channels get "pushed" up strongly, making then approximtely equal in magnitude to the dominant channel, leading to a graying out of the overall region. The MSR produces a much better final image in terms of color, and dynamic range than the SSR. The differences are easier to see in the original color images (Fig. 7 a-f and 8).

\section{Performance metrics}

The PSNR block computes the peak signal-to-noise ratio, in noise, between two images between original image and enhancement image (Kaur \& Singh, 2014). The higher PSNR gives the better quality of an enhanced image. The Mean Square Error (MSE) and the Peak Signal to Noise Ratio (PSNR) are the two error metrics used to compare image-enhancement quality. The MSE represents the cumulative squared error between an enhancement 

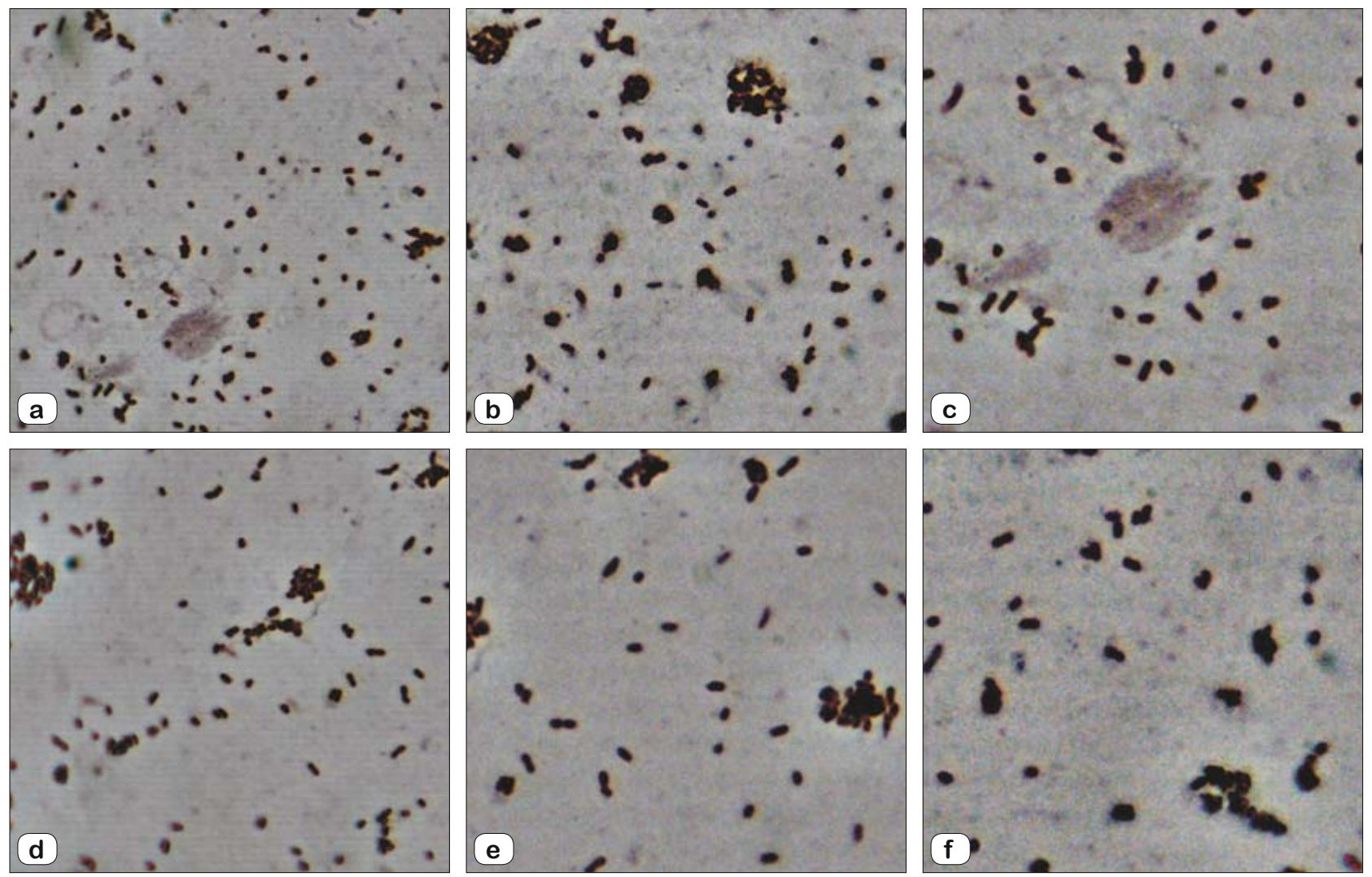

Fig. 7 (a-f). Multiscale color image enhancement result.

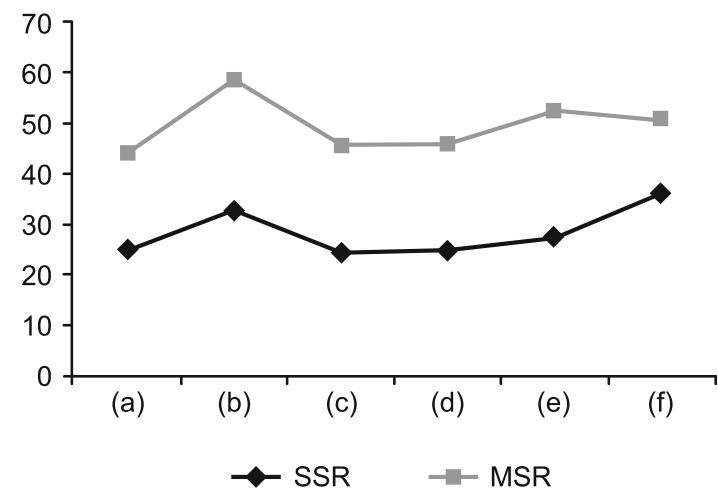

Fig. 8. Performance chart of the Color enhancement filters.

image $\left(I_{2}\right)$ and the original image $\left(I_{1}\right)$, whereas PSNR represents a measure of the peak error. To compute the PSNR, the block first calculates the mean-squared error using the following equation:

$$
M S E=\frac{\sum_{M, N}\left[I_{1}(m, n)-I_{2}(m, n)\right]^{2}}{M N}
$$

In the previous equation, $M$ and $N$ are the number of rows and columns in the input images, respectively. Then the block computes the PSNR using the following equation:

$$
P S N R=10 \log _{10}\left(\frac{R^{2}}{M S E}\right)
$$

In the previous equation, $R$ is the maximum fluctuation in the input image data type. For example, if the input image has a double-precision floating-point data type, then $R$ is 1 . If it has an 8-bit unsigned integer data type, $R$ is 255 . It means maximum possible grey level values in the image.

\section{Result and discussion}

In this paper, Performance evaluation of different filters is done by PSNR parameters. Higher in PSNR values show that the particular filter is performing well. From the results shown in Table 1 , it is observed that PSNR values for median filter are high when compared to wiener filter. On average, it produces a PSNR value of $49.50 \mathrm{db}$. Similarly, the same filter produces low PSNR values when compared to other filters. From the results shown in Table 2, it is observed that PSNR values for MSR filter is high when compared to SSR filter. On average, it produces a PSNR value of $49.43 \mathrm{db}$. So overall, Median filter is superior filter to other filters in greyscale image enhancement and MSR filter is superior filter to other filters in color image enhancement.

This work presents an investigation on the analysis of pneumonia bacteria images. We studied how the use of bacteria images 
may improve the quality of image. We have shown results using actual digital microscopic pneumonia bacteria images. We found that a filtering strategy based on greyscale image enhancement tool and color image enhancement tool allows the enhancement of bacteria images. Based on input images and grayscale image enhancement technique best performance results are given by the Median filter and color image enhancement technique best performance results are given by the Multiscale retinex technique. A very important task for the future is the development of smarter and more detailed enhancement schemes.

\section{References}

1. Hogeweg P. The roots of bioinformatics in theoretical biology. PLoS Comput Biol 2011; 7 (3): e1002021.

2. Hesper B, Hogeweg P. Bioinformatica: een werkconcept. Kameleon 1970; 1 (6): 28-29.

3. Hogeweg P. Simulating the growth of cellular forms. Simulation 1978; 31 (3): 90-96.

4. Pathak SS, Dahiwale P, Padole G. A combined effect of local and global method for contrast image enhancement. in Engineering and Technology (ICETECH), 2015 IEEE International Conference on, 2015, 1-5.

5. Cepeda-Negrete J, Sanchez-Yanez RE. Automatic selection of color constancy algorithms for dark image enhancement by fuzzy rule-based reasoning. Appl Soft Comput 2015; 28: 1-10.

6. Anbarjafari G, Jafari A, Jahromi MNS, Ozcinar C, Demirel H. Image illumination enhancement with an objective no-reference measure of illumination assessment based on Gaussian distribution mapping. Eng Sci Technol Int J 2015; 18 (4): 696-703.

7. D’liez S, Sánchez S. Algorithms for contrast enhancement of electronic portal images. Radiat Phys Chem 2015; 116: 14-20.

8. Kumar G, Murugan D, Kavitha R, Manish TI. New information technology of performance evaluation of road extraction from high resolution satellite images based on PCNN and CV model. Informatologia 2014; 47 (2-3): 121-134.
9. Atta R, Abdel-Kader RF. Brightness preserving based on singular value decomposition for image contrast enhancement. Opt J Light Electron Opt 2015; 126 (7): 799-803.

10. Cho D, Bui TD. Fast image enhancement in compressed wavelet domain. Signal Processing 2014; 98: 295-307.

11. He W, Feng W, Peng Y, Chen Q, Gu G, Miao Z. Optik Multi-level image fusion and enhancement for target detection. Opt Int J Light Electron Opt 2015; 126 (11-12): 1203-1208.

12. Manish TI, Murugan D, Kumar TG. Edge detection by combined Canny filter with scale multiplication \& ant colony optimization. in Proceedings of the Second International Conference on Computational Science, Engineering and Information Technology, 2012, 497-500.

13. Manish TI, Murugan D, Kumar GT. Hybrid edge detection using canny and ant colony optimization. Commun Inf Sci Manag Eng 2013; 3 (8): 402.

14. Kumar TG, Murugan D, Manish TI. Comparative Analysis on Road Extraction from Liss IV Near-Infrared Band Using Genetic Algorithm and Active Contour Model.

15. Yu Y, Wu T. Image enhancement using the averaging histogram equalization ( AVHEQ ) approach for contrast improvement and brightness preservation q. Comput Electr Eng 2015; 1-15.

16. Kumar TG, Murugan D, Rajalakshmi K, Manish TI. Image enhancement and performance evaluation using various filters for IRS-P6 Satellite Liss IV remotely sensed data. Geofizika 2015; 32 (2): 179-189.

17. Martin MP, Samarasinha N, Larson S. CometCIEF : A web-based image enhancement facility to digitally enhance images of cometary comae. Planet Space Sci 2015; 118: 181-186.

18. Wu C, Liu Z, Jiang H. Catenary image enhancement using waveletbased contourlet transform with cycle translation. Optik (Stuttg) 2014; 125 (15): 3922-3925.

19. Mastin GA. Adaptive filters for digital image noise smoothing: An evaluation. Comput Vision Graph Image Process 1985; 31 (1): 103-121.

20. Arias-Castro E, Donoho DL. Does median filtering truly preserve edges better than linear filtering? Ann Stat 2009; 1172-1206.

21. Davies ER. Machine Vision: Theory, Algorithms, Practicalities. 1997. Academic Press, 1997. 